\title{
Cell line and drug-dependent effect of ERBB3 on cancer cell proliferation, chemosensitivity, and multidrug actions
}

This article was published in the following Dove Press journal:

International Journal of High Throughput Screening

24 April 2010

Number of times this article has been viewed

\section{Bo Chen \\ Rui Mao \\ Hongjie Wang \\ Jin-Xiong She'}

'Center for Biotechnology and Genomic Medicine, Department of Pathology; Medical College of Georgia, Augusta, GA, USA
Correspondence: Jin-Xiong She

Center for Biotechnology and Genomic Medicine, Medical College of Georgia, II 20 I5th Street, Augusta, GA, 30912, USA

$\mathrm{Tel}+\mathrm{I} 7067213410$

Fax +I 7067213688

Email jshe@mail.mcg.edu
Purpose: The purpose of this study is to investigate the potential role of ERBB3 in cancer cell proliferation, chemosensitivity, and multidrug actions. ERBB3, also called Her3 (human epidermal growth factor receptor 3 ) in humans, is a type I transmembrane glycoprotein that is a member of the ERBB family of tyrosine kinase receptors.

Methods: Cancer cell proliferation was assessed in three different cancerous cell lines (Hela, $\mathrm{CM}$, and MCF7) transfected with the control or ERBB3 siRNA (small inhibitory ribonucleic acid, a short sequence of RNA which can be used to silence gene expression). A high-throughput screening (HTS) system with siRNA knockdown was developed to investigate the effect of ERBB3 on chemosensitivity and multidrug actions of United States Food and Drug Administration (FDA) approved drugs.

Results: ERBB3 knockdown by siRNA significantly reduces the proliferation of Hela cells but not CM or MCF7 cells. Among the 30 tested FDA-approved anticancer drugs, all three types of cancer cells (Hela, CM, and MCF) are completely killed by nine common drugs and are completely resistant to seven common drugs. Furthermore, Hela, CM, and MCF7 cells are sensitive to six, nine, and two additional drugs, respectively, while they are partially sensitive to four, four, and one other drugs, respectively. ERBB3 knockdown enhances the sensitivity of Hela cells to seven drugs and only enhances the sensitivity of MCF cells to one drug (aclarubicin). In contrast, ERBB3 knockdown does not enhance chemosensitivity of CM cells to any tested drugs, but desensitizes these cells to erlotinib treatment. Our results also indicate that troglitazone (TRG), a peroxisome proliferator-activated receptor (PPAR $\gamma$ ) agonist and potentially useful agent to reduce multiple drug resistance, potently inhibits the proliferation of Hela but not MCF7 or CM cells. Furthermore, TRG and other drugs have complex interactions that depend, at least partially, on the expression levels of ERBB3.

Conclusion: The effect of ERBB3 on cancer cell proliferation, chemosensitivity, and multidrug actions depends on the types and combination of cancer cells and drugs. Therefore, when ERBB3 is targeted for cancer therapy, careful consideration must be paid to each patient's individualized characteristics to select the most beneficial drug or drug combination.

Keywords: ERBB family, anticancer drugs, siRNA, drug resistance, high-throughput screening, FDA-approved drugs

\section{Introduction}

ERBB is a family of epidermal growth factor receptor (EGFR) tyrosine kinases, which regulate signaling for the maintenance of cell division, proliferation, differentiation, migration, and other normal cell processes. ${ }^{1}$ ERBB3, a unique member of EGFR family, is a glycoprotein of $180 \mathrm{kDa}$ which consists of an extracellular domain followed by a transmembrane-spanning helix and an intracellular cytoplasmic kinase domain. 
Unlike other ERBB receptors, ERBB3 lacks intrinsic tyrosine kinase activity and cannot autophosphorylate due to the evolutionary acquisition of several changes within the kinase domain. ${ }^{2,3}$ The primary ligands for ERBB3 are the members of the neuregulins (NRG) family. After NRG activation, the tyrosine residue of the kinase domain of ERBB3 is transactivated by physical association with other ERBB family members, to form highly potent heterodimers or higher-order oligomers. ${ }^{4}$ ERBB3 may also be transactivated by cellular stress and cytokines including tumor necrosis factor $\alpha$ (TNF- $\alpha$ ) and interferon $\alpha$ (INF- $\alpha){ }^{5}$ Activated ERBB3 has the highest binding affinity for phosphoinositol 3-kinase (PI3K) among the ERBB receptors. ${ }^{6,7}$ Consequently, activation of ERBB3 downstream prominently reacts with PI3K/ AKT, a strong mitogenic signal.

In addition to regulating signaling for normal cell processes, accumulating evidence has suggested that ERBB3 plays a critical role in cancer and other diseases. Overexpression of ERBB3 has been frequently detected in a variety of cancers, including those of the breast, colon, stomach, ovary, and pancreas. ${ }^{8-16}$ ERBB3 is implicated in coupling ERBB1 to the PI3K/ AKT pathway in nonsmall cell lung cancers (NSCLCs) that are sensitive to EGFR inhibitors such as gefitinib. ${ }^{17}$ The PI3K/AKT signal is a critical oncogenic stimulus whose aberrant activity leads to apoptosis resistance in a wide range of cancers. ${ }^{5,18}$ In ERBB2-driven cancers, ERBB3 functions as an intimate signaling partner that promotes the transforming potency of ERBB2, usually by activating the PI3K/AKT pathway. Intestine-specific ERBB3 ablation resulted in almost complete absence of intestinal tumors in the $\mathrm{Apc}^{\mathrm{Min}}$ mouse model of colon cancer, ${ }^{2}$ suggesting that the ERBB3 pseudo-kinase has essential roles in supporting intestinal tumorigenesis (Apc ${ }^{\mathrm{Min}}$ [Min = multiple intestinal neoplasia $]$ is a point mutation in the murine homolog of the APC gene). Therefore, ERBB3 may be a promising target for the treatment of cancers. Smallinterfering RNA (siRNA) to ERBB3 recently tested in vivo appears to have significant reducing effects on human lung adenocarcinoma. ${ }^{19}$ Recent evidence has suggested that ERBB3 is also responsible for tumor resistance to therapeutic agents. It may contribute to tamoxifen-resistance in breast cancer cells. ${ }^{20-22}$ There are compelling results showing that ERBB3 activity up-regulation is a means of escape from therapeutic suppression by several tyrosine kinase inhibitors in breast cancer. ${ }^{21,22}$ In our present study, we investigated the potential impact of ERBB3 on cancer proliferation, sensitivity to a large number of United States Food and Drug Adminstration (FDA) approved drugs and drug interactions using a high-throughput screening (HTS) approach.

\section{Material and methods \\ Reagents}

Human ERBB3 siRNA and control siRNA were purchased from Santa Cruz Biotechnology (Santa Cruz, CA). All antibodies used for fluorescent activated cell sorting (FACS) analysis were from R\&D system (Minneapolis, MN). Reagents for intracellular staining were purchased from eBioscience (San Diego, CA). Cell counting kit-8 (CCK-8) was purchased from Dojindo Molecular Technologies, Inc (Rockville, MD).

\section{Cell culture and siRNA knockdown}

MCF7 and Hela cell lines were obtained from the American Type Culture Collection (ATCC, Rockville, MD). The CM cell line is a human insulinoma cell line kindly provided by Dr Palo Pozzilli at the London School of Medicine and Dentistry. ${ }^{23,24}$ All the cell lines were grown at RPMI 1640 medium containing $10 \%$ fetal bovine serum (complete medium) and cultured in a $37^{\circ} \mathrm{C}$ humidified atmosphere containing $95 \%$ air and $5 \% \mathrm{CO}_{2}$. Cells were split twice a week. Specific ERBB3 siRNA or control siRNA were transfected into cultured cells with Lipofectamine ${ }^{\mathrm{TM}} 2000$ reagent (Invitrogen, Carlsbad, CA) according to the procedures provided by manufacture. Cells were used for assays 24 hours after siRNA transfection.

\section{Drug library and screening}

A library of 640 FDA-approved drugs was purchased from BIOMOL International (Philidelphia, PA, USA). All BIOMOL compounds are selected to maximize chemical and pharmacological diversity, and have known and well-characterized bioactivities. One-hundred nanoliters of each drug (at a final concentration of $2 \mu \mathrm{g} / \mathrm{mL}$ ) was robotically transferred into $50 \mu \mathrm{L}$ of culture medium in 96-well plates. Cancer cells were then added into the wells at a density of 5,000 cells/well.

\section{FACS analysis}

Cells were incubated with human $\mathrm{Fc}$ fragment for $15 \mathrm{~min}$ at $4{ }^{\circ} \mathrm{C}$ then labeled for $30 \mathrm{~min}$ at $4{ }^{\circ} \mathrm{C}$ with antibodies against human EGFR, ERBB2, ERBB3, ERBB4, or isotype controls (R\&D system). For intracellular staining, cells were permeabilized according to the manufacturers' instructions using intrastaining kit (eBioscience) followed by staining with anti-human ERBB3 antibody for $30 \mathrm{~min}$ at $4{ }^{\circ} \mathrm{C}$. Thereafter, cells were washed and resuspended in phosphatebuffered solution (PBS) with $0.1 \%$ fetal bovine serum (FBS). Fifty thousand cells were collected and analyzed using 
FACSCalibur (BD Biosciences, San Jose, CA) and the FACS CellQuest pro software (version 5.0.1; BD Biosciences).

\section{Cell proliferation assay}

Cell count kit- 8 was used to determine cell viability. Briefly, after 72 hours of culture, reagents were added to the plates and the plates were read at $490 \mathrm{~nm}$ with a microplate reader (Nexcelom, Lawrence, MA). Cell numbers were derived by comparing optic density of the experimental wells to a cell standard with known cell numbers. Cell viability (percentage of surviving cells) was calculated by comparing cell numbers in experimental groups to the controls, which are defined as having $100 \%$ cell viability. All experiments were repeated three times. Means and standard deviations were presented in the figures. Statistical tests were performed using two-sided Student's $t$-test.

\section{Results}

\section{ERBB3 promotes Hela cell proliferation}

Since up-regulation of ERBB family members (EGFR, ERBB2, ERBB3, and ERBB4) has been frequently observed in many types of tumors, we examined their surface expression in three cancer cell lines: Hela, MCF-7, and CM. All four ERBBs were expressed on MCF7 and CM cells
(Figure 1A). However, ERBB3 was not detectable on the surface of Hela cells (Figure 1A). Since previous studies showed that the ERBB3 protein is predominantly localized in the cytoplasm of ovarian cancer cells, ${ }^{25}$ we performed intracellular staining for ERBB3 and found weak levels of intracellular ERBB3 in Hela cells (Figure 1B).

To investigate the potential role of ERBB3 in cancer cell proliferation, we determined the proliferation rates of the cancer cell lines after transfection with ERBB3 siRNA or control siRNA. The ERBB3 siRNA can efficiently knockdown ERBB3 (Figure 1C). Cell proliferation assays indicate that ERBB3 knockdown significantly inhibited the proliferation of Hela cells $(P<0.0001)$, but had no effect on the proliferation of MCF7 or CM cells (Figure 2). Previous studies have already shown that ERBB3 knockdown did not alter the proliferation of MCF7 cells ${ }^{21}$ and another pancreatic cancer cell line. ${ }^{26}$ Taken together, our results suggest that ERBB3 knockdown reduces cell proliferation only in certain types of cancer cells.

\section{ERBB3 knockdown largely sensitizes, but can desensitize, cancer cells to treatment}

To assess the effect of different drugs on the proliferation/survival of the three cancer cell lines, the cells were treated with 160 FDA-approved drugs (arrayed on two 96-well plates)
A

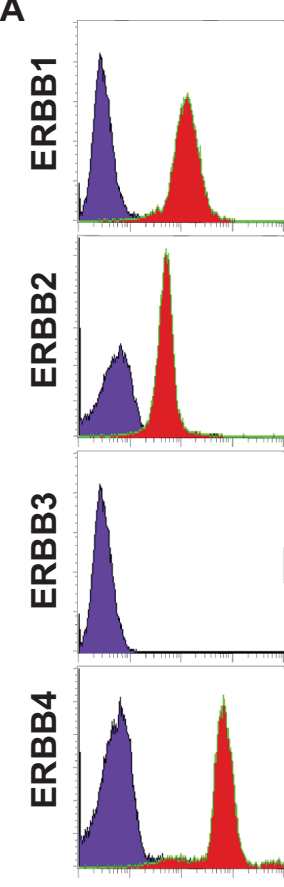

Hela
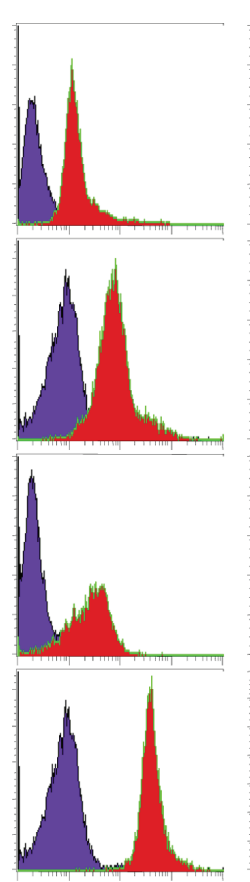

CM
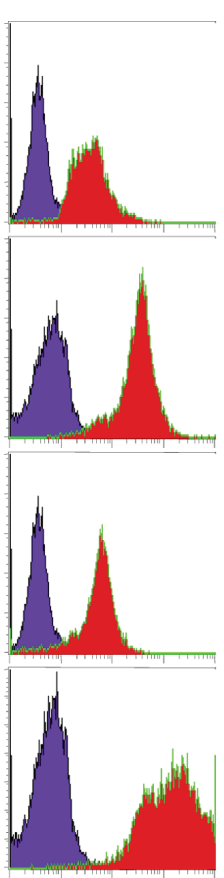

MCF-7
B

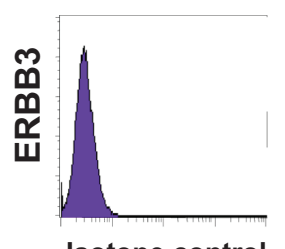

Isotope control

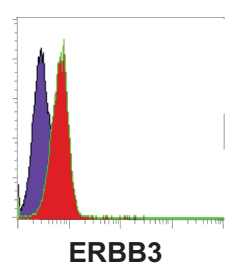

C
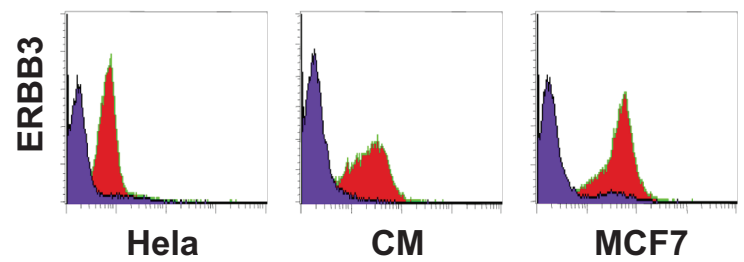

ERBB3 knockdown, intracellular staining

Figure I Expression of ERBB family members in cancer cell lines. A) Fluorescent activated cell sorting (FACS) analysis of surface ERBB expression in three cancer cell lines. Blue $=$ isotype control, red $=$ ERBB family member labeled on the left of the panels. B) Intracellular staining for ERBB3 in Hela cells. Blue $=$ isotype control, red $=$ ERBB3. C) Intracellular staining of ERBB3 protein after knockdown by ERBB3 siRNA (blue) or control siRNA (red). 


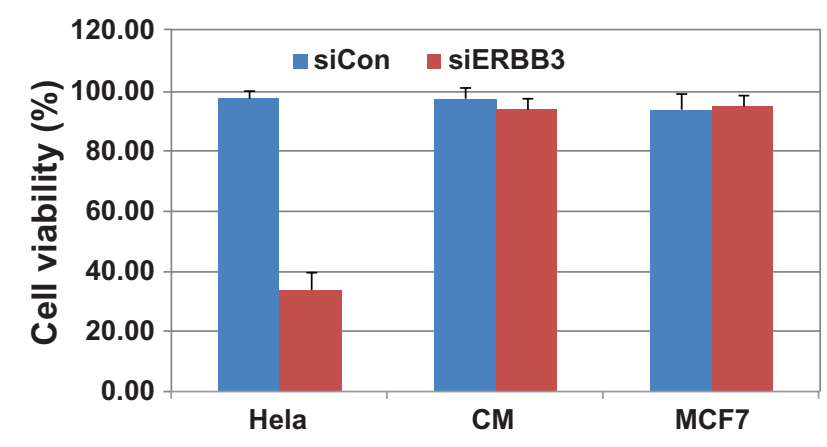

Figure 2 ERBB3 knockdown reduces Hela cell proliferation. Three different types of cancer cells were transfected with control siRNA (siCon) or ERBB3 siRNA (siERBB3). Twenty-four hours post-transfection, transfected cells were plated onto 96-well plates and cultured for 72 hours, and cell numbers were then measured with the CCK- 8 kit. The means and standard deviations of relative cell viability are calculated from three independent experiments.

including 30 anticancer drugs. As shown in Figure 3, these cells have different chemosensitivity to anticancer drugs. CM and Hela cells appear to be sensitive to more drugs compared to MCF7 cells. CM cells are sensitive to 18 of the 30 drugs, partially sensitive to four other drugs, and resistant to eight drugs (Figure 3). Hela cells are sensitive to 15 drugs, partially sensitive to four drugs and resistant to 11 drugs. MCF7 cells are sensitive to only 11 drugs and partially sensitive to another drug but resistant to the remaining 18 drugs. It is interesting to note that all three lines are sensitive to nine common drugs and resistant to seven common drugs. The sensitivity for the remaining 14 drugs differs between cell lines.

To determine the potential impact of ERBB3 on the chemosensitivity of cancer cells to different drugs, we treated the three cancer cell lines transfected with ERBB3 or control siRNA and compared cell viability. ERBB3 had no detectable impact on drug sensitivity for 22 of the 30 cancer drugs studied here. Figure 4 presents data on the remaining eight drugs which have differential efficacy depending on ERBB3 expression in at least one cell line. ERBB3 knockdown increases the sensitivity of Hela cells to four drugs (camptothecin, hydroxycamptothecin, tanshinone IIA, and gemcitabine) $(P=0.003)$, while its effect on the 8th drug (aclarubicin) cannot be assessed because it completely killed Hela cells in our assay conditions. ERBB3 knockdown enhances the sensitivity of MCF7 cells to only one drug (aclarubicin) $(P=0.007)$. Interestingly, ERBB3 knockdown does not enhance sensitivity of $\mathrm{CM}$ cells to any drug but desensitizes $\mathrm{CM}$ cells to the tyrosine kinase inhibitor erlotinib $(P=0.0007)$ (Figure 4).

\section{ERBB3 knockdown has variable effect on the action of drug combinations}

Troglitazone (TRG) is a peroxisome proliferator-activated receptor (PPAR $\gamma$ ) agonist that is capable of downregulating multiple drug resistance protein (MDR-1) and restoring sensitivity of MCF7 cells to drug treatment. ${ }^{27}$ Therefore TRG is being explored as a drug to overcome drug resistance. Our results indicate that TRG is a potent inhibitor of Hela cell proliferation, while it has no impact on the proliferation of MCF7 and CM cells (Figure 5). Interestingly, when TRG is combined with other drugs, Hela cells are not completely killed (Figure 6A), suggesting a complex interaction between TRG and other drugs. The interaction between TRG and other drugs also depends on the expression levels of ERBB3 (Figure 6). TRG enhances the activ-

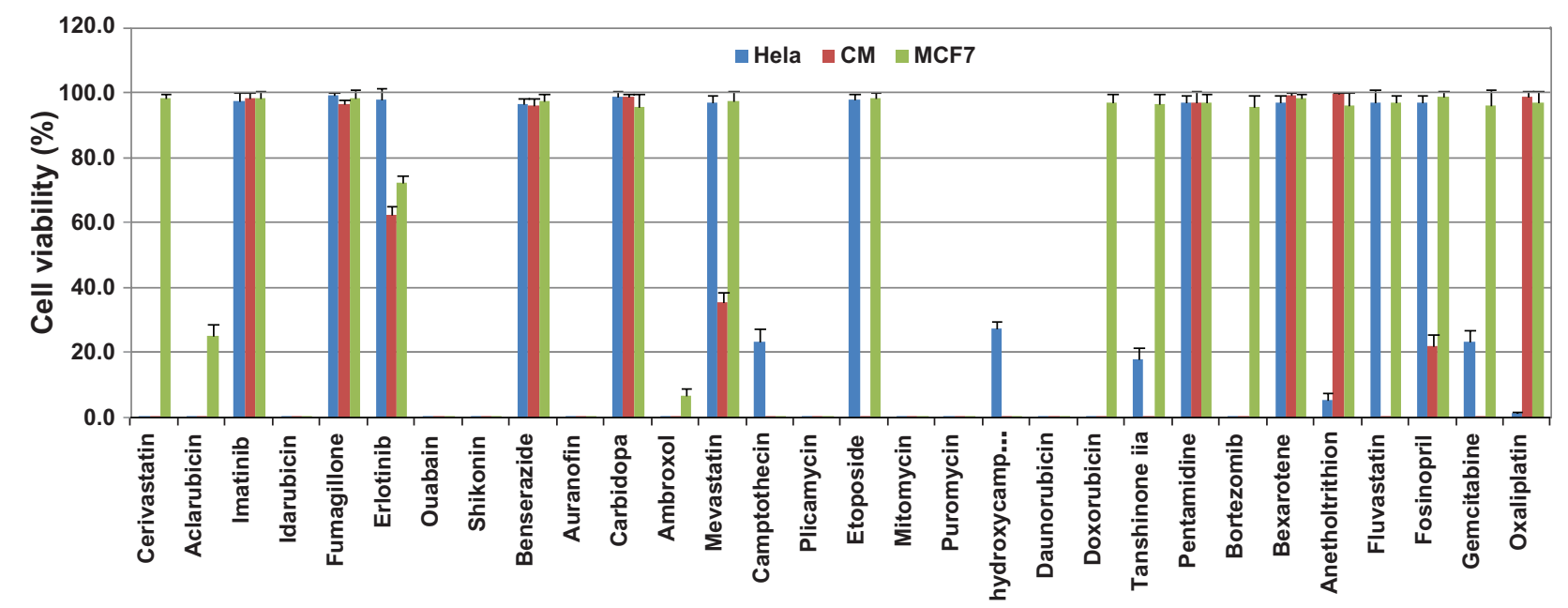

Figure 3 Efficacy of 30 anticancer drugs on the proliferation of three cancer cell lines. Identical number (5,000/well) of cells was plated onto 96 -well plates and cultured with one of the 160 FDA-approved drugs for 72 hours. The means and standard deviations of relative cell viability are calculated from three independent experiments. Results for 30 anticancer drugs are presented here. Hydroxycamptothecin $=10$-hydroxycamptothecin. 


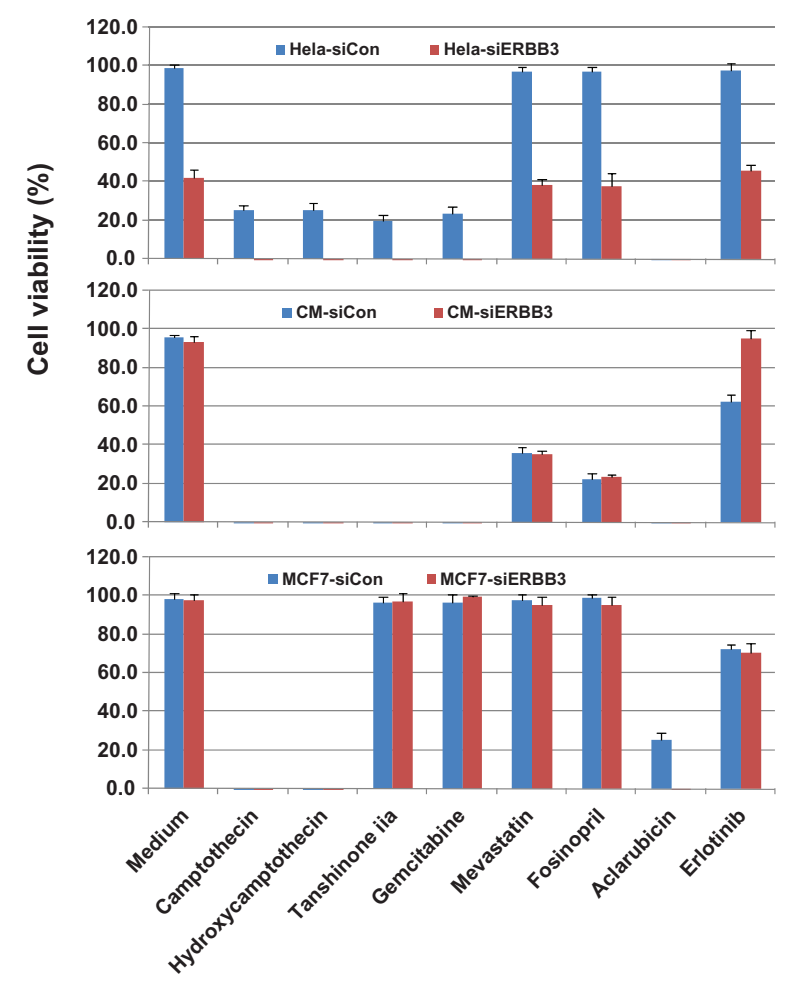

Figure 4 ERBB3 knockdown modulates chemosensitivity of some cancer drugs. Three different types of cancer cells were transfected with control siRNA (siCon) or ERBB3 siRNA (siERBB3). Twenty-four hours post-transfection, transfected cells were plated onto 96-well plates and cultured for 72 hours, and cell numbers were measured with the CCK- 8 kit. The means and standard deviations of relative cell viability are calculated from three independent experiments.

ity of 10-hydroxyxamptothecin and tanshinone IIA, but decreases the activity of camptothecin and gemcitabine, while it has no major effect on the activity of mevastatin, fosinopril, and erlotinib to ERBB3 siRNA-treated Hela cells (Figure 6B).

\section{Discussion}

Two of the ERBB family members, EGFR and ERBB2, have been extensively targeted for cancer drug development. EGFR is being targeted with the monoclonal antibody cetuximab and with two small molecule tyrosine kinase inhibitors, gefitinib and erlotinib. ${ }^{28}$ ERBB2 has been successfully targeted by the monoclonal antibody trastuzumab (Herceptin ${ }^{\circledR}$ ) in breast cancers, where it is often overexpressed. ${ }^{29}$ In contrast, efforts at targeting ERBB3 in cancers have lagged behind, due in part to its impaired kinase activity. ${ }^{30}$ It has becoming increasingly clear that ERBB3 plays a critical role in cancer progression. Intestine-specific ERBB3 ablation resulted in almost complete absence of intestinal tumors in the APC ${ }^{\text {Min }}$ mouse model of colon cancer. ${ }^{2}$ A siRNA-based therapy has also been attempted in lung cancer. ${ }^{19} \mathrm{~A}$ number of previous studies have addressed the importance of ERBB3 in cancer cell proliferation. It has become apparent that the role of ERBB3 depends on the other ERBB family members. ${ }^{31,32}$ For example, overexpression of EGFR and ERBB3 in NIH3T3 cells does not result in tumorigenesis, while overexpression of ERBB2 and ERBB3 does. ${ }^{33,34}$ This study suggests that knockdown of ERBB3 may not necessarily alter the proliferation ability of two tumor cell lines (MCF7 and CM) that have high levels of surface expression of all 4 ERBB proteins. Similar results have already been published for MCF7 cells. ${ }^{21}$ In contrast, knockdown of ERBB3 in the Hela cells, which have high levels of surface EGFR, ERBB2, and ERBB4, but no detectable surface ERBB3, dramatically reduces Hela cell proliferation. The mechanism underlying the different outcome of ERBB3 on cell proliferation is still unclear and deserves further investigation.

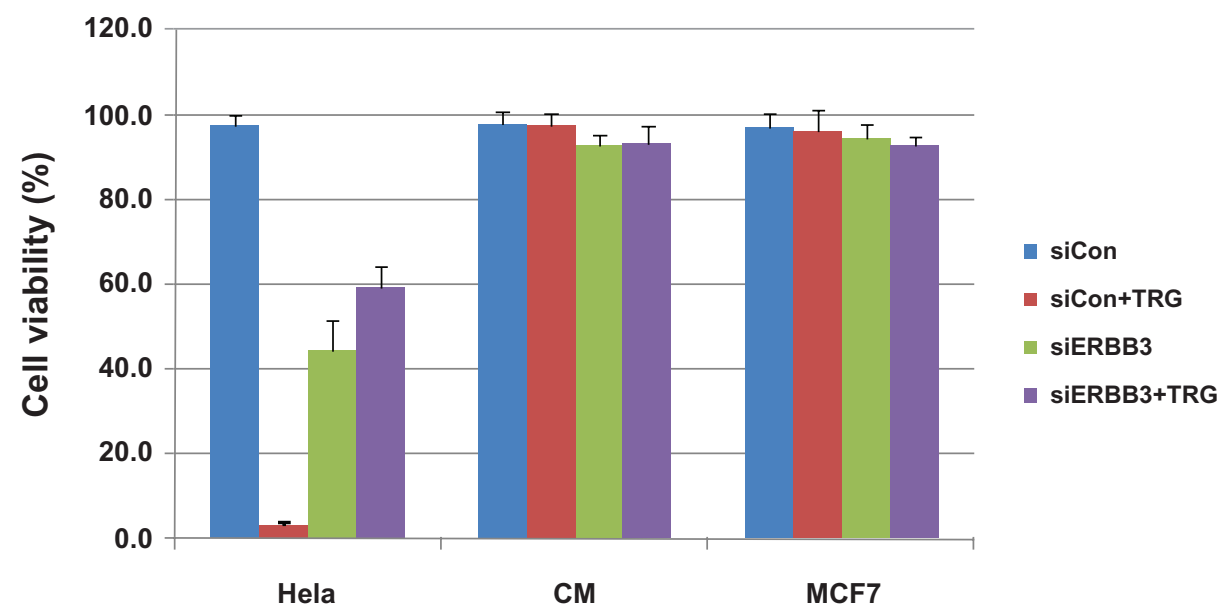

Figure 5 Troglitazone (TRG) inhibits Hela cell proliferation in an ERBB3-dependent manner. Three different types of cancer cells were transfected with control siRNA (siCon) or ERBB3 siRNA (siERBB3). Twenty-four hours post transfection, transfected cells were plated onto 96 -well plates and cultured for 72 hours, and cell numbers were measured with the CCK-8 kit. The means and standard deviations of relative cell viability are calculated from three independent experiments. 


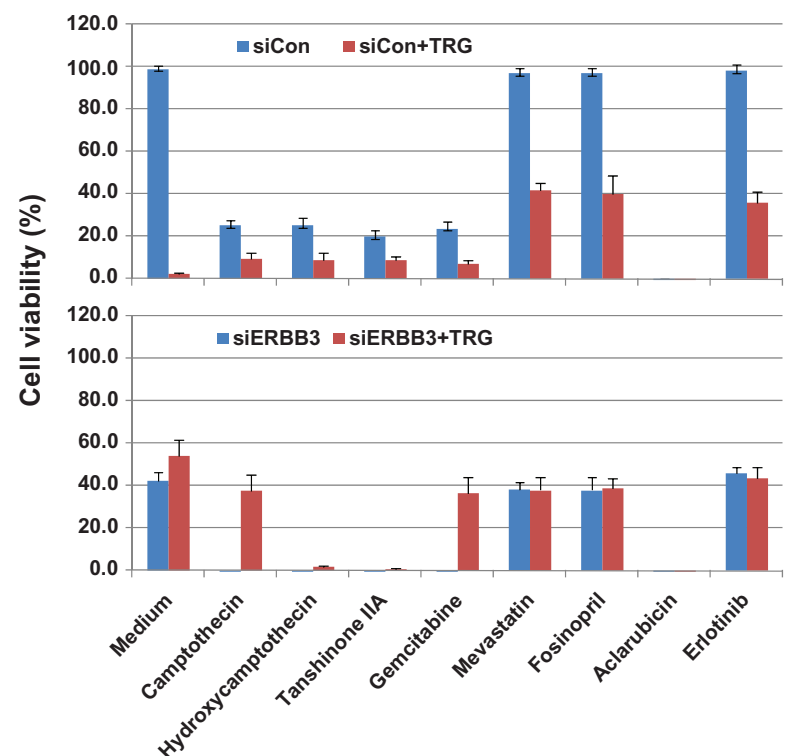

Figure 6 Differential effects of ERBB3 on multidrug actions in Hela cells. A) Treatment of Hela cells transfected with control siRNA by troglitazone (TRG), or TRG plus one of the eight drugs at the bottom of the figure. Hela cells were transfected with control siRNA. Drugs were added 24 hours post-transfection and cell numbers were measured 72 hours postdrug treatment. Experiments were identical to B) except that ERBB3 siRNA was used.

Our studies demonstrate that MCF7 cells are resistant to more cancer drugs than both CM and Hela cells (Figure 3). Consistent with the impact of ERBB3 in Hela cell proliferation, knockdown of ERBB3 significantly sensitizes Hela cells to the treatment with 15 anticancer drugs. Even though ERBB3 does not appear to be essential to the proliferation of MCF7 cells, ERBB3 knockdown does sensitize MCF7 cells to aclarubicin but not to other cancer drugs tested in this study. These results suggest that ERBB3 may be an important factor that influences the chemosensitivity in some cancers. One of the most interesting findings of this study is the observation that ERBB3 knockdown can actually desensitize some cancer cells to treatment with a single drug or a combination of drugs, suggesting that the outcome of targeting ERBB3 for cancer therapy may depend on the types of cancers and drugs.

\section{Conclusion}

The effect of ERBB3 on cancer proliferation, chemosensitivity, and multidrug actions depends on the types and combination of cancer cells and drugs. When ERBB3 is targeted for cancer therapy, careful consideration must be paid to each patient's individualized characteristics to select the most beneficial drug or drug combination.

\section{Disclosures}

The authors report no conflicts of interest in this work.

\section{References}

1. Leahy DJ. Structure and function of the epidermal growth factor (EGF/ ErbB) family of receptors. Adv Protein Chem. 2004;68:1-27.

2. Lee D, Yu M, Lee T, et al. Tumor-specific apoptosis caused by deletion of the ERBB3 pseudo-kinase in mouse intestinal epithelium. J Clin Invest. 2009;119:2702-2713.

3. Mei L, Xiong WC. Neuregulin 1 in neural development, synaptic plasticity and schizophrenia. Nat Rev Neurosci. 2008;9:437-452.

4. Yarden Y, Sliwkowski MX. Untangling the ERBB signalling network. Nat Rev Mol Cell Biol. 2001;2:127-137.

5. Yen L, Benlimame N, Nie ZR, et al. Differential regulation of tumor angiogenesis by distinct ERBB homo- and heterodimers. Mol Biol Cell. 2002;13:4029-4044.

6. Soltoff SP, Carraway KL III, Prigent SA, Gullick WG, Cantley LC. ErbB3 is involved in activation of phosphatidylinositol 3-kinase by epidermal growth factor. Mol Cell Biol. 1994;14:3550-3558.

7. Kim HH, Sierke SL, Koland JG. Epidermal growth factor-dependent association of phosphatidylinositol 3-kinase with the ERBB3 gene product. J Biol Chem. 1994;269:24747-24755.

8. Naidu R, Yadav M, Nair S, Kutty MK. Expression of C-ERBB3 protein in primary breast carcinomas. Br J Cancer. 1998;78:1385-1390.

9. Ciardiello F, Kim N, Saeki T, et al. Differential expression of epidermal growth factor-related proteins in human colorectal tumors. Proc Natl Acad Sci U S A. 1991;88:7792-7796.

10. Maurer CA, Friess H, Kretschmann B, et al. Increased expression of ERBB3 in colorectal cancer is associated with concomitant increase in the level of ERBB2. Hum Pathol. 1998;29:771-777.

11. Kobayashi M, Iwamatsu A, Shinohara-Kanda A, Ihara S, Fukui Y. Activation of ERBB3-PI3-kinase pathway is correlated with malignant phenotypes of adenocarcinomas. Oncogene. 2003;22:1294-1301.

12. Jackson-Fisher A, Bellinger G, Breindel J, et al. ERBB3 is required for ductal morphogenesis in the mouse mammary gland. Breast Cancer Res. 2008;10:R96.

13. Hayashi M, Inokuchi M, Takagi Y, et al. High expression of HER3 is associated with a decreased survival in gastric cancer. Clin Cancer Res. 2008;14:7843-7849.

14. Koutras AK, Kalogeras KT, Dimopoulos MA, et al. Evaluation of the prognostic and predictive value of HER family mRNA expression in high-risk early breast cancer: A Hellenic Cooperative Oncology Group (HeCOG) study. Br J Cancer. 2008;99:1775-1785.

15. Lin SH, Lee YC, Choveiri MB, et al. Soluble ERBB3 levels in bone marrow and plasma of men with prostate cancer. Clin Cancer Res. 2008; 14:3729-3736.

16. Sithanandam G, Anderson LM. The ERBB3 receptor in cancer and cancer gene therapy. Cancer Gene Ther. 2008;15:413-448.

17. Engelman JA, Janne PA, Mermel C, et al. ERBB3 mediates phosphoinositide 3-kinase activity in gefitinib-sensitive non-small cell lung cancer cell lines. Proc Natl Acad Sci U S A. 2005;102:3788-3793.

18. Rajkumar T, Stamp GW, Hughes CM, Gullick WJ. C-ERBB3 protein expression in ovarian cancer. Clin Mol Pathol. 1996; 49:M199-M202.

19. Johnson JI, Decker S, Zaharevitz D, et al. Relationships between drug activity in NCI preclinical in vitro and in vivo models and early clinical trials. Br J Cancer. 2001;84:1424-1431.

20. Sergina NV, Rausch M, Wang D, et al. Escape from HER-family tyrosine kinase inhibitor therapy by the kinase-inactive HER3. Nature. 2007;445:437-441.

21. Liu B, Ordonez-Ercan D, Fan Z, et al. Downregulation of ERBB3 abrogates ERBB2-mediated tamoxifen resistance in breast cancer cells. Int J Cancer. 2007; 120:1874-1882.

22. Sergina NV, Moasser MM. The HER family and cancer: emerging molecular mechanisms and therapeutic targets. Trends Mol Med. 2007; 13:527-534.

23. Cavallo MG, Pozzilli P, Misiti S, Baroni MG. Insulinoma CM cell line as in vitro model for beta-cell. $J$ Cell Physiol. 2008;216:568. 
24. Cavallo MG, Dolta F, Monetini L. Beta-cell markers and autoantigen expression by a human insulinoma cell line: similarities to native beta cells. J Endocrinol. 1996;150:113-120.

25. Tanner B, Hasenclever D, Stevn K, et al. ERBB3 predicts survival in ovarian cancer. J Clin Oncol. 2006;24:4317-4323.

26. Frolov A, Schuller K, Tzeng CW, et al. ERBB3 expression and dimerization with EGFR influence pancreatic cancer cell sensitivity to erlotinib. Cancer Biol Ther. 2007;6:548-554.

27. Davies GF, Juurlink BH, Harkness TA. Troglitazone reverses the multiple drug resistance phenotype in cancer cells. Drug Des Devel Ther. 2009;3:79-88.

28. Mendelsohn J, Baselga J. Epidermal growth factor receptor targeting in cancer. Semin Oncol. 2006;33:369-385.

29. Yeon CH, Pegram MD. Anti-ERBB2 antibody trastuzumab in the treatment of HER2-amplified breast cancer. Invest New Drugs. $2005 ; 23: 391-409$
30. Sithanandam G, Anderson LM. The ERBB3 receptor in cancer and cancer gene therapy. Cancer Gene Ther. 2008;15:413-448.

31. Harari D, Tzahar T, Romano J, et al. Neuregulin-4: a novel growth factor that acts through the ERBB-4 receptor tyrosine kinase. Oncogene. 1999; 18:2681-2689.

32. Pinkas-Kramarski R, Shelly M, Guarino BC, et al. ERBB tyrosine kinases and the two neuregulin families constitute a ligand-receptor network. Mol Cell Biol. 1998;18:6090-6101.

33. Nakano N, Higashiyama S, Kajihara K, et al. NTAKalpha and beta isoforms stimulate breast tumor cell growth by means of different receptor combinations. J Biochem. 2000;127:925-930.

34. Hijazi MM, Young PE, Dougherty MK, et al. NRG-3 in human breast cancers: activation of multiple ERBB family proteins. Int J Oncol. 1998;13:1061-1067.
International Journal of High Throughput Screening

\section{Publish your work in this journal}

International Journal of High Throughput Screening is an international, peer-reviewed, open access journal publishing original research, reports, editorials, reviews and commentaries dedicated to all aspects of high throughput screening, especially related to drug discovery and associated areas of biology and chemistry. The manuscript management sys-

\section{Dovepress}

tem is completely online and includes a very quick and fair peer-review system. Visit http://www.dovepress.com/testimonials.php to read real quotes from published authors.

Submit your manuscript here: http://www.dovepress.com/international-journal-of-high-throughput-screening-journal 